IAU Colloquium 164: Radio Emission from Galactic and Extragalactic Compact Sources

ASP Conference Series, Vol. 144, 1998

J. A. Zensus, G. B. Taylor, \& J. M. Wrobel (eds.)

\title{
Water in NGC 1068
}

\section{J. Greenhill}

Harvard-Smithsonian Center for Astrophysics, Cambridge, MA 02138, U.S.A.

Abstract. We report the first VLBI images of the NGC $1068 \mathrm{H}_{2} \mathrm{O}$ maser that include the full $600 \mathrm{~km} \mathrm{~s}^{-1}$ spread of the emission. The structure of the source is suggestive of an edge-on massive disk that is probably bound by the central engine of this AGN.

\section{Introduction}

There are about 20 water maser sources known to lie in active galactic nuclei (AGN). The masers may be associated with 1) accretion disks and supermassive central engines or 2) jets. The well studied maser source in NGC 4258 is the archetype for the first type of maser, and the maser source in the Seyfert 2 nucleus of NGC 1068 may be a second example.

Images of the NGC 4258 maser strongly suggest a thin annulus of molecular gas that is between 0.13 and $0.25 \mathrm{pc}$ from the AGN central engine (Miyoshi et al. 1995 ) and that that is perpendicular to the axis of the radio jet associated with the AGN (Herrnstein et al. 1997). Maser emission close to the systemic velocity of the galaxy arises from material on the near side of the disk. High-velocity emission that is red and blue-shifted by $\pm(800-1100) \mathrm{km} \mathrm{s}^{-1}$ arises on the disk diameter perpendicular to the line of sight. These masers sample a Keplerian rotation curve $\left(v \propto r^{-0.5}\right)$ with deviations of $<1 \%$.

Water maser emission in NGC 1068 is associated with radio component $\mathrm{S} 1$, which lies at the base of a roughly north-south radio jet (Gallimore et al. 1996). The maser extends about $\pm 300 \mathrm{~km} \mathrm{~s}^{-1}$ from the systemic velocity (Figure 1). Two early VLBI observations resolved structure within the red-shifted and systemic emission (Gwinn et al. 1993; Greenhill et al. 1996) but were not sensitive to the blue-shifted emission.

The first VLBI observation to cover the $600 \mathrm{~km} \mathrm{~s}^{-1}$ spread of the maser clearly shows that the maser source is linear, extending over $\sim 2$ pc from the red-shifted to the blue-shifted extreme (Figure 2). The source may be described approximately by an edge-on disk with rotation velocities of up to $330 \mathrm{~km} \mathrm{~s}^{-1}$, somewhat similar to the source in NGC 4258. The maser features trace the disk over radii $0.65<r<1.1 \mathrm{pc}$. The enclosed mass for $r<0.65 \mathrm{pc}$ is $\approx 1.7 \times 10^{7} \mathrm{M}_{\odot}$ and the inferred Eddington ratio $\left(L / L_{E}\right)$ is $\approx 25 \%$, for $L=4.4 \times 10^{44} \mathrm{erg} \mathrm{s}^{-1}$ (Pier et al. 1994). The rotation curve is sub-Keplerian (i.e., more shallow than $\left.v \propto r^{-0.5}\right)$, which is consistent with a disk mass that is $\approx 8 \%$ of the central mass, where we have assumed a radial density distribution that is appropriate for an $\alpha$ accretion disk (Figure 3 ). The predicted accretion rate and disk thickness are $0.03 \alpha^{1.1} \mathrm{M}_{\odot} \mathrm{yr}^{-1}$ and $110 \alpha^{.07} \mu$ as, respectively. The disk may be warped by about $10^{\circ}$ in position angle and inclination.

The orientation and dimensions of the model molecular disk are interesting in light of jet and molecular emission on scales of $10-100 \mathrm{pc}$. The position angles of the axes of the radio jet, the nuclear bar traced in $\mathrm{CO}$, and the rotation axis of the innermost portion of the disk differ by only $\sim 20^{\circ}$. Moreover, velocity gradients in CO (Helfer \& Blitz 1995) and HCN (Tacconi et al. 1994) emission 

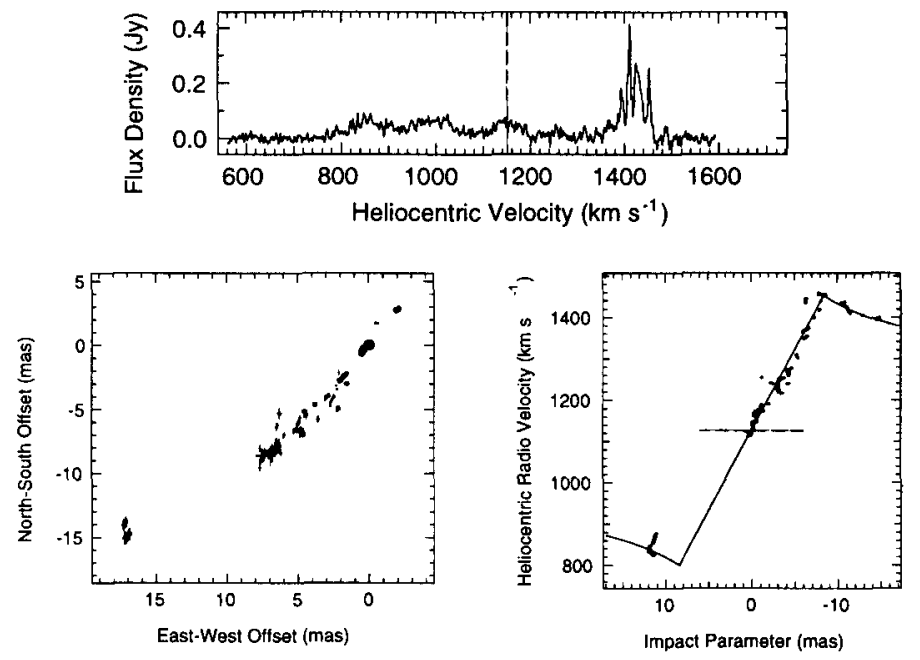

Figure 1. (top)-Spectrum of the NGC $1068 \mathrm{H}_{2} \mathrm{O}$ maser. The dashed line indicates a systemic velocity estimated from $\mathrm{H}$ I emission (Brinks et al. 1997). (left)-Distribution of maser emission. At a distance of $15 \mathrm{Mpc}, 0.7 \mathrm{pc}$ subtends 10 mas. (right)-Positionvelocity diagram. Impact parameter is measured with respect to systemic emission at $(+7,-8)$ mas (positive to the northwest). The rough symmetry in position and velocity and the declining rotation curve at large radii are characteristics of a rotating edge-on disk.

are almost perpendicular to the disk axis. The inner radius of the disk is approximately the radius at which dust sublimates. The disk inner diameter is also approximately the size of the hot ionized source resolved by Gallimore et al. (1997) and inferred to be part of the obscuring torus. These observations and the observations of the maser disk provide direct evidence of the structures and significant column densities along the line of sight to the central engine of NGC 1068.

\section{References}

Brinks, E., et al. 1997. Ap\&SS, 248, 23-31.

Gallimore, J. F., Baum, S. A., O'Dea, C. P. 1997. Nature, 388, 852-854.

Gallimore, J. F., et al. 1996. ApJ, 462, 740-745.

Greenhill, L. J., et al. 1996. $A p J, 472, \mathrm{~L} 21-24$.

Gwinn, C. R., et al. 1993. In Sub-Arcsecond Radio Astronomy, eds. R. J. Davis \& R. S. Booth (Cambridge: Cambridge University Press), 331-332.

Helfer, T. T., \& Blitz, L. 1995. ApJ, 450, 90-110.

Herrnstein, J. R., et al. 1997. $A p J, 475$, L17-21.

Miyoshi, M., et al. 1995. Nature, 373, 127-130.

Pier, E. A., et al. 1994. ApJ, 428, 124-129.

Tacconi, L. J., et al. 1994. $A p J, 426$, L77-80. 